\title{
An uncontrolled manifold analysis of arm joint variability in virtual planar position and orientation tele-manipulation
}

\author{
J. Buzzi, Student Member, IEEE, E. De Momi Member, IEEE, and I. Nisky, Member, IEEE
}

\begin{abstract}
Objective: In teleoperated robot-assisted tasks, the user interacts with manipulators to finely control remote tools. Manipulation of robotic devices, characterized by specific kinematic and dynamic proprieties, is a complex task for the human sensorimotor system due to the inherent biomechanical and neuronal redundancies that characterize the human arm and its control. We investigate how master devices with different kinematics structures and how different task constraints influence users capabilities in exploiting arm redundancy. Methods: A virtual teleoperation workbench was designed and the arm kinematics of seven users was acquired during the execution of two planar virtual tasks, involving either the control of position only or position-orientation of a tool. Using the UnControlled Manifold Analysis of arm joint variability we estimated the logarithmic ratio between task irrelevant and the task relevant manifolds $\left(R_{v}\right)$. Results: The $\mathbf{R}_{\mathbf{v}}$ values obtained in the position-orientation task were higher than in the position only task while no differences were found between the master devices. A modulation of $R_{v}$ was found through the execution of the position task and a positive correlation was found between task performance and redundancy exploitation. Conclusion: Users exploited additional portions of arm redundancy when dealing with the tool orientation. The $R_{v}$ modulation seems influenced by the task constraints and by the users possibility of reconfiguring the arm position. Significance: This work advances the general understanding of the exploitation of arm redundancy in complex tasks, and can improve the development of future robotic devices.
\end{abstract}

Index Terms-Redundancy exploitation, teleoperation, uncontrolled manifold (UCM)

\section{INTRODUCTION}

$\mathbf{N}$ OWADAYS, teleoperated robotic systems are employed in many different fields, ranging from handling of hazardous materials, dangerous area explorations, precise manufacturing and surgery. Among the other applications of this technology, teleoperated Robot-Assisted Minimally Invasive Surgery (RAMIS) has seen a substantial growth over the last

This work was supported in part by the Helmsley Charitable Trust through the ABC Robotics Initiative, by the Marcus Endowment Fund both at Ben-Gurion University of the Negev, and the ISF grant number $823 / 15$.

J. Buzzi \& E. De Momi are with the Department of Electronics, Information and Bioengineering at the Politecnico di Milano, 20133 Milan, Italy (email: jacopo.buzzi@polimi.it)

I. Nisky is with the Department of Biomedical Engineering and with Zlotowski Center for Neuroscience, Ben-Gurion University of the Negev, Be'er Sheva, 84105, Israel decade thanks to its several advantages with respect to standard minimally invasive surgery (MIS) [1]. While teleoperating, users (i.e. surgeons) interact with master devices to control slave instruments that directly interact with the environment (i.e. tissue). This architecture offers improvements compared to open and standard techniques, such as high precision thanks to hand motion scaling and tremor filtering. In addition, it provides teleoperators with an ergonomic and comfortable setup, and wristed robotic tools allow for high dexterity and an intuitive mapping of hand to end-effector movements [2]-[5].

Multiple studies focused on the analysis of the relation between teleoperators and master devices, and explored different control methods ranging from keyboards, robotic devices, and optical hand tracking; these studies monitored clinically relevant performance metrics [6]-[8]. However, teleoperation represents a complex motor control task, and forces the users to acquire new skills to fully take advantage of this technology [9]-[11]. The human motor control system is characterized by multiple degrees of redundancy (kinematic, kinetic, sensory and neuronal). This redundancies allow for one of the characteristics of a healthy human movement: variability, which translates into multiple unique neural and motor patterns to solve the same motor task [12]. Instead of freezing the arm joints in the same solutions, experts exploit this redundancy, and use this variability in their arm joints to increase performance. To develop intuitive teleoperation systems and controllers, and to further understand redundancy exploitation in the human arm, it is beneficial to model the exploitation of arm kinematic and kinetic redundancy in different teleoperation and manipulation conditions [13], [14].

Multiple studies tried to assess how the Central Nervous System (CNS) solves the ill-posed problems of inverse kinematics and inverse dynamics by reducing degrees of freedom [15]. The most common approach to solve the redundancy has been the definition of kinematic and kinetic cost functions that guide the CNS in planning the best joint trajectories [16]-[18]. In these models, the inherent redundancy is solved by replacing the behavioral goal with the specific desired trajectory that better fits the cost function. These models require as input a detailed description of the unique and planned joint trajectories predefined by the CNS. However, the existence of variable equally valid solutions to multiple tasks involving motor redundancy, exposed the limit of these models [19].

To account for this variability in the kinematic solutions, 
Todorov and Jordan proposed optimal feedback control as a theory of movement coordination. In this framework, the optimal solution is achieved online with a feedback control that uses all the time-varying feedback available to correct only those deviations that interfere with the task goals [20]. A goaloriented performance criterion together with a regularization term that minimizes control effort is defined, and the movement details are automatically filled by searching the control policy that achieves the best performance [21]. Similarly, Gelfand and Latash defined the principle of abundance for which families of solutions are generated by the CNS by covarying elemental variables so that no differences are induced in the output task space [22]. , Instead of being considered as a burden they proposed the role of motor redundancy in achieving high performance while minimizing the control effort [23].

Several methods were proposed to quantify the level of redundancy exploitation by evaluating the variability distribution in geometrically defined subspaces [24]-[26]. The Tolerance Noise Covariation (TNC) principle describes how humans cope with the neuromotor noise and decompose variability into exploitation of task tolerance, stochastic noise, and covariation between variables. In the TNC, the Task Irrelevant Subspace, or Manifold (TIM), is entirely defined in the task space and, therefore, it requires an analytical model of the task dynamics. Thanks to the task dependent definition of the TIM, the TNC has been used to estimate how the three components of variance change during the learning process [24]. Another performance analysis of redundancy exploitation is represented by the Goal Equivalent Manifold (GEM) [25], [26]. The GEM is presented as the set of possible task solution strategies that arise from the theoretical definition of a goal function. Through this analysis, the sensitivity of task space errors to joint space perturbations can be estimated along with the degree of alignment between body variability and the GEM. Similarly to the TNC, the GEM is defined in the task space, thus requiring an in-depth knowledge of the task dynamics.

A powerful tool for the estimation of redundancy exploitation when the task dynamics is unknown and an analytical model that describes the task space is absent, is the Uncontrolled Manifold (UCM) analysis [27]. In this framework, the TIM definition is based on the average experimental control variables trajectories, allowing the extension of performance evaluation to those fields where there is no a-priori knowledge of the task goal. Within the UCM framework, in accordance with the Minimum Intervention Principle (MIP) [20], humans control and limit the variability aligned with the task related manifold (TRM) while leaving unrestrained the task-irrelevant (TIM) variability that has no effect on the task performance. For linear systems, the TIM can be computed as the null space of the matrix that relates control variables to task variables. For nonlinear systems, an approximation of the TIM can be computed as the null space of the linearized relation between control variables and task variables. Instead of analytical linearization, a linear relation can also be estimated from sampled data by means of linear regression approximation [28].

Recently, Sternad et al. [29] demonstrated that the UCM analysis is sensitive to the choice of the coordinates that are used to define the task related and unrelated manifolds. An elegant solution to this problem was recently proposed [30], but it was only applied to static tasks to date. In this study, we focus on dynamic tasks in which the structure of the GEM is not well-defined. Therefore, in spite of its limitations, we chose to employ the UCM, and we discuss the limitations in depth in the discussion section.

The UCM analysis was adopted to study a large variety of tasks such as reaching movements [27], bimanual pointing [31], stone knapping [32] and isometric finger force exertion [33]. Recently, it was employed in robotic teleoperation [34] to discriminate between expert and novice teleoperators and investigate the effect of teleoperation manipulator dynamics on redundancy exploitation. Nisky et al. showed how expert teleoperators exploited their arm kinematic redundancy more than novices and especially when teleoperating. However, this study only focused on simple point-to-point reach movements, and compared a single teleoperation manipulator to freehand.

In RAMIS, many tasks involve movement along curved paths and a control of the orientation of the master manipulator and the surgical instruments [35]-[37]. Quantifying the control of orientation was recently shown to be promising for assessment of surgical skills [36], [37]. Only few previous studies analyzed how tasks that require control of orientation affected motor redundancy exploitation using the UCM approach, [38], [39]. For example, Morrison et al. recently showed how golfers used different levels of redundancy exploitation through the golf swing execution, and that they favored the control of the golf clubhead orientation rather than its position. The control of orientation during fine manipulation tasks is yet to be fully understood, and in the studies that focused on orientation, the redundancy exploitation that is associated with the position or orientation are analyzed in isolation [31], [37]-[41]

In this study, to address the aforementioned gaps, we employ the UCM framework to analyze how the characteristics of the task, in form of different levels of curvature, may influence the coordination of variability in the control variables throughout the tasks execution. We also seek to understand how users prioritized the control of position and orientation in a combined path and orientation tracing task. We designed two tracing in virtual teleoperation. The tasks involve different task space dimensions and natures: we compared the exploitation of redundancy during the execution of a two-dimensional planar position tracing with the exploitation of redundancy during the execution of a three-dimensional planar task, in which both the position and the orientation of a virtual tool had to be controlled.

Our hypotheses are the following:

(HP1) Users may maximize the task redundancy exploitation in face of high complexity portions of the trajectory.

(HP2) The mechanical, dynamic and work space differences between a hybrid parallel/serial link (Sigma) and a serial link (Omni) master device would modify novice users' capabilities of exploiting the arm redundancy.

(HP3) Mixed position-orientational task constraints may influence users capabilities in exploiting arm redundancy. 
A.

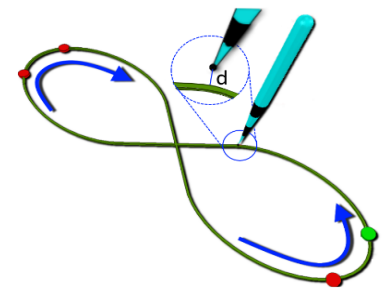

B.

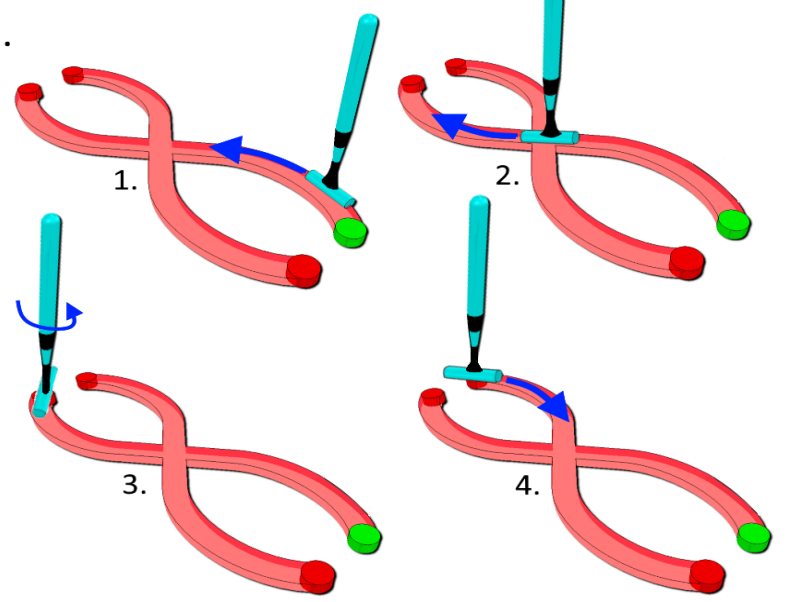

Fig. 1. The two virtual tasks that were developed. $\ln \boldsymbol{T}_{\mathbf{2} D}(\mathrm{~A})$ users were required to follow an eight-shaped path controlling a virtual stylus tip. In $\boldsymbol{T}_{3} \boldsymbol{D}$ (B), users were also required to control the tool's orientation throughout the path

\section{MethodS}

\section{A. Tasks design}

Two tasks were developed in a virtual reality scenario (Vrep, Coppelia Robotics, Zurich, Switzerland) to challenge users with different task goals and complexities. Users were asked to precisely follow a path at a self defined comfortable speed (tracing task) and, for both tasks, an eight-shaped path was chosen. The path was modeled as half of the cloverleaf motions presented by Levit-Binnun et al. [42].

1) Task $2 \boldsymbol{D}\left(T_{2 D}\right)$ : Users were requested to follow the path with a virtual stylus tool (Fig. 1A). They started from an initial position (green dot in Fig. 1A) and moved first counter-clockwise, then straight crossing the intersection in the middle, finally in a clock-wise direction to return to the starting position. To compensate for the absence of tridimensional depth perception, the path color turned green when the tool-tip of the stylus was within \pm $20 \mathrm{~mm}$ from the plane where the trajectory lied or red otherwise. Users were required to precisely minimize the $x-y$ distance between the tool tip and the path while avoiding to excessively stray way from the task plane. Since an intuitive and immediate visual feedback was present for the $x-y$ errors only, due to the lack of 3D perception, only these task dimensions were considered in the UCM analysis $\left(d_{t a s k, T_{2 D}}=2\right.$, see Fig. 2.B).

2) Task $3 D\left(T_{3 D}\right)$ : Users were required to follow the same path while also orienting the cylindrical tool tip along the path, as shown in Fig. 1B. The tool end-effector had to slide over the shape fitted path starting from the green dot (Fig. 1.B.1). Due to the structure of the master devices (see Section II-B), to twist the virtual tool, users had to rotate their wrist while maintaining fingers' grip over the handle of the devices fixed. After flexing the wrist until the central intersection was reached (Fig. 1.B.2), users had to extend it to reach the top left. At the upper open end of the path (red dot in (Fig. 1.B.2), the users had to reposition the tool end-effector on the shape fitted path (see Fig. 1.B.3). Similarly, the users had to reach the lower open end (Fig. 1.B.4) to complete the task.

The same color feedback as in $T_{2 D}$ was adopted. In $T_{3 D}$ the task space is composed of two positional $(\mathrm{x}, \mathrm{y})$ and one rotational $(\alpha)$ element, therefore $d_{t a s k, T_{3 D}}=3$ (see Fig. 2.B).

\section{B. Master devices}

The virtual teleoperation tasks were implemented using two master devices: a 6+1 DoFs hybrid parallel/serial link haptic interface (Sigma) and a 6 DoFs serial links haptic interface (Omni).

1) Hybrid Parallel/Serial master device (Sigma): The Force Dimension Sigma7 (Force Dimension, Nyon, Switzerland) was used as Sigma and it's characterized by six degrees of freedom plus an active grasping control (see Fig. 2.A.2). The master device is gravity compensated, has a resolution of $0.0015 \mathrm{~mm}$ and $0.013 \mathrm{deg}$ and its workspace can be approximated to an elliptical dome with radiuses of $190 \times 130 \mathrm{~mm}$. The master device controller, based on a Linux API, sampled the gripper position and orientation at $1 \mathrm{kHz}$.

2) Serial Link master device (Omni): A Phantom Omni (3D Systems, South Carolina, USA) was used as Omni (see Fig. 2.A.3). Controlled by a stylus end effector, within the parallelepipedal workspace of $160 \times 120 \times 70 \mathrm{~mm}$, the device is characterized by a resolution of $0.055 \mathrm{~mm}$. Omni's position and orientation not gravity compensated controller ran at $500 \mathrm{~Hz}$.

\section{Acquisition framework}

The kinematics of the user was acquired using two localization devices: the thorax position was acquired using an optical localization system (see Fig. 2.A.4) (Vicra, Northern Digital, Ontario, Canada, $20 \mathrm{~Hz}$ sampling rate, $0.25 \mathrm{~mm}$ position Root Mean Squared Error) using three passive retroreflective markers attached to the right and left acromions and next to the jugular notch (see Fig. 2.A.5).

The arm, forearm and hand positions were acquired using an electromagnetic localization system (see Fig. 2.A.6) (Aurora, Northern Digital, Ontario, Canada, $30 \mathrm{~Hz}$ sampling rate, 0.48 $\mathrm{mm}$ and $0.3 \mathrm{deg}$ position and orientation RMSE, dome shaped field with a radius of approximately $500 \mathrm{~mm}$ ) and three 6-DoF $1.8 \times 9 \mathrm{~mm}$ electromagnetic sensors (see Fig. 2.A.7).

The electromagnetic 6-DoF markers were used to generate 2 virtual markers for each body segment and they were placed at recognizable body landmarks calibrated on the users' elbow, wrist and hand in a pre-acquisition phase. The two acquisition systems were then registered to the same reference frame using an Hand Eye calibration approach [43]. 

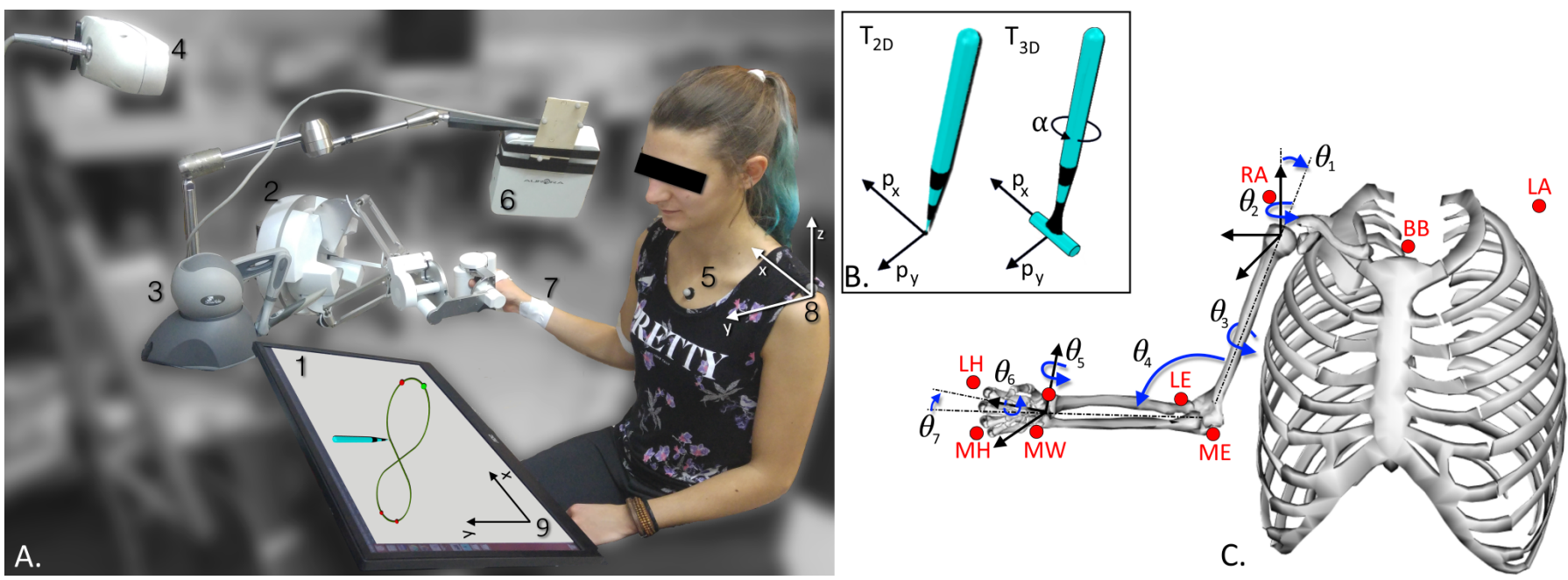

Fig. 2. A. The experimental setup: user performs the tasks while looking at a flat screen (1) and teleoperating with the hybrid parallel/serial link master device (Sigma - 2) or using the serial link master device (Omni - 3). The thorax movements are recorded using an optic camera (4) with three retroreflecting markers (5) while the arm kinematics is acquired with an electromagnetic tracker (6) and 6DoFs markers (7). The shoulder reference frame (8) is grossly aligned with the VR one (9). B. In the magnifying box, $\boldsymbol{T}_{\mathbf{2} D}$ and $\boldsymbol{T}_{\mathbf{3} \boldsymbol{D}}$ task spaces. C. OpenSim model used for the inverse kinematics. In blue the seven degrees of freedom $(1,27)$ and in red the virtual markers set-up: Left and Right Acromium (LA, RA), Breast Bone (BB) for the thorax, Lateral and Medial Elbow (LE, ME) for the arm, Lateral and Medial Wrist for the forearm (LW, MW), Lateral and Medial Hand (LH, MH) for the hand orientation.

\section{Experimental protocol}

Seven healthy right handed users were recruited (4 female and 3 male, mean age $23 \pm 1.5$ y.o.) and provided informed written consent in accordance with the Declaration of Helsinki.

The users sat in front of a desk, where a 2D monitor (see Fig. 2.A.1) was laid as flat as possible to be approximately parallel to the plane in which the tasks were performed (see Fig. 2.A.9). The seat position and height were adjusted for each user to achieve the best personal comfort and interface with the master devices. Users were instructed to find their own trade-off between precision and execution speed during the execution of the tasks, that were performed with both master devices for each user.

For each user, the experiment order was randomized so that users performed 10 repetitions of a randomly selected experiment before moving to the next one.

\section{E. Joint angle estimation}

The users' arm was modeled as three rigid segments and 7 degrees of freedom. The shoulder joint was modeled as a ball and socket joint, comprising abduction-adduction, flexionextension and external-internal rotation of the forearm. The elbow was modeled as a single flexion-extension DoF while the wrist joint consists in three DoFs: pronation-supination, flexion-extension, abduction-adduction [44].

To estimate the arm joint configuration, OpenSim's inverse kinematics and a widely adopted upper limb musculoskeletal model were adopted [45]-[47]. Raw marker movements were filtered (recursive 2nd order Butterworth filter with cut-off frequency $6 \mathrm{~Hz}$ ) and static acquisitions were used to scale the model to fit the users' arm and thorax dimensions. The offline inverse kinematics algorithm takes as input each frame of the acquired markers positions and finds the best joint vector $\mathrm{q}$ that minimizes the error between the experimental and virtual marker positions:

$$
\min _{\mathbf{q}}\left[\sum_{k=1}^{K}\left\|\mathbf{p}_{k}^{a c q}-p_{k}^{v i r t}(\mathbf{q})\right\|^{2}\right]
$$

With $\boldsymbol{q}$ vector of joint angles, $\mathbf{p}_{k}^{a c q}$ is the acquired 3D position of the $k^{t h}$ marker, $\mathbf{p}_{k}^{v i r t}(\mathbf{q})$ is the position of the corresponding virtual marker on the model that depends on the vector of joint angles $\boldsymbol{q}$ and $K$ is the number of markers.

\section{F. Uncontrolled Manifold Analysis}

To analyze the coordination between the arm joint angles and the task execution, we adopted the Uncontrolled Manifold Analysis (UCM) [48]. To evaluate the stability of joint angles around the time-varying reference path, we first computed the trial-to-trial variability around an average path. We assume that, for each user, task, and master device, the same arm state is defined by the CNS as a kinematic strategy to follow the space-normalized path [49]. While we do not know what the real reference path is, and whether such reference exists in the motor system, for the sake of the analysis, these reference joint and task space trajectories were estimated from the betweentrials average path.

First, to account for the between-user and between-trial variability in the task execution speed, the kinematic data from the inverse kinematic algorithm $\left(\theta_{u, n, m, r}\right)$ was spacenormalized using the curvilinear abscissa of the path $[s]$. All the task trials among the different users were re-sampled at the same number of equally-spaced samples that was equal to the number of frames of the shorter trial. Then, the between-trials average trajectories and the variability around them could be computed. Therefore, the total joint-space variance $V_{\theta_{u, n, m}}$ is 
defined as:

$$
V_{\theta_{u, n, m}}[s]=\sum_{r=1}^{N}\left\|\theta_{u, n, m, r}[s]-\bar{\theta}_{u, n, m}[s]\right\|^{2} d_{\text {joints }}^{-1} N^{-1}
$$

where $\theta_{u, n, m, r}[\mathrm{~s}]$ represents the joint angles vector for the $u^{\text {th }}$ user $(u=1: U, U=7), n^{\text {th }}$ task $\left(n=T_{2 D}, T_{3 D}\right)$, $m^{\text {th }}$ master device $\left(m=\right.$ Sigma,Omni), $r^{\text {th }}$ trial $(r=1$ : $N, N=10)$ at the $s^{\text {th }}$ space-normalized sample ( $\left.=0: 1\right)$ solution of the inverse kinematics algorithm, and $\bar{\theta}_{u, n, m}[\mathrm{~s}]$ represents the average (across the $\mathrm{N}$ repetitions of the same task) joint angles vector at the $s^{\text {th }}$ space-normalized sample. The joint-space dimension $d_{\text {joints }}=7$ is the number of joints in our model of the arm.

For small deviations from the average path, the joint kinematics can be linearized using the Jacobian matrix $J(\theta[s])$ that relates joint velocities $\dot{\theta}=\left[\dot{\theta_{1}}, \dot{\theta_{2}}, \dot{\theta_{n}}\right]$ to the end-effector Cartesian $(\mathrm{x}, \mathrm{y}, \mathrm{z})$ and angular $(\gamma, \beta, \alpha)$ velocities $\dot{x}$ :

$$
\dot{\mathbf{x}}=\mathbf{J}(\boldsymbol{\theta}) \dot{\boldsymbol{\theta}}
$$

To obtain a linear approximation of the TIM, the null space of the Jacobian at each average configuration was computed.

$$
J\left(\overline{\boldsymbol{\theta}}_{u, n, m}[s]\right) \cdot \boldsymbol{\epsilon}=0
$$

Where $\epsilon$ is the basis vectors matrix, whose dimension is $d_{T I M}=d_{\text {joints }}-d_{\text {task }}$ and $\overline{\boldsymbol{\theta}}_{u, n, m}[s]$ is the mean joint trajectory defined as follows.

$$
\overline{\boldsymbol{\theta}}_{u, n, m}[s]=\frac{1}{N} \sum_{r=1}^{N} \boldsymbol{\theta}_{u, n, m, r}[s]
$$

Using the null space basis vector matrix $\epsilon$ it's possible to project the deviations from the average joint path onto the null space:

$$
\boldsymbol{\theta}_{T I M_{u, n, m, r}}[s]=\boldsymbol{\epsilon} \boldsymbol{\epsilon}^{T}\left(\boldsymbol{\theta}_{u, n, m, r}[s]-\overline{\boldsymbol{\theta}}_{u, n, m}[s]\right)
$$

and onto the orthogonal task-relevant manifold (TRM):

$$
\boldsymbol{\theta}_{T R M_{u, n, m, r}}[s]=\left(\boldsymbol{\theta}_{u, n, m, r}[s]-\overline{\boldsymbol{\theta}}_{u, n, m}[s]\right)-\boldsymbol{\theta}_{T I M_{u, n, m, r}}[s]
$$

In the tracing task presented, the TRM can be visualized as a combination of joint angle variations that affect task performance, generating movements of the tool's end-effector. The TIM is composed of joint angle variations that will leave task performance unaltered by leaving the position of the tool end-effector unchanged. Importantly, while geometrical orthogonality is not defined between joint angle variations, if the linearization holds true, a mathematical orthogonal manifold can always be extracted.

Similarly to Eq. 2, it's possible to obtain the variance associated to the two projections:

$$
\begin{aligned}
& V_{T I M_{u, n, m}}[s]=\sum_{r=1}^{N}\left\|\theta_{T I M_{u, n, m, r}}[s]\right\|^{2} d_{\text {TIM }}^{-1} N^{-1} \\
& V_{T R M_{u, n, m}}[s]=\sum_{r=1}^{N}\left\|\theta_{T R M_{u, n, m, r}}[s]\right\|^{2} d_{\text {task }}^{-1} N^{-1}
\end{aligned}
$$

Where $d_{\text {task }}$ is the task space dimension which represents the number of Cartesian and angular degrees of freedom that the users are required to control to perform the task and depends on the task type. To estimate the users' capabilities in exploiting the arm redundancy, without being biased by the overall joint-space variance, the logarithmic ratio between the two variances $R_{v}[s]$ is computed:

$$
R_{v}[s]=\log \left(\frac{V_{T I M}[s]}{V_{T R M}[s]}\right)
$$

When $R_{v}$ is positive, users' variability along the TIM is higher than $V_{T R M}$, showing that users' are exploiting arm redundancy.

\section{G. Jacobian estimation}

When the model of the arm is available, the Jacobian matrix can be obtained from the concatenation of the homogeneous matrices that define the forward kinematics and their derivation. In this study, we use the user specific scaled model obtained through OpenSim to obtain the analytical expression of the Jacobian. To apply the UCM analysis, it is necessary to reduce the dimension of the full rank Jacobian to account for the task space dimensions that users were trying to control.

The Jacobian is computed with respect to the shoulder reference frame, which is aligned with the task space frame. Therefore, to extract the task space positional components in the $x-y$ plane (see Fig. 2B), it's possible to extract the corresponding Jacobian rows. On the other hand, the rotation that users were required to control (see Fig. 2B) is defined in the end effector reference frame and corresponds to the tool pitch angle. With respect to the shoulder reference frame, depending on the arm configuration, this angle might have components in all the three rows of the Jacobian rotational part, consequently, it would be less intuitive to extract the angle only. The Jacobian was therefore also estimated with a regression method from the joint angle variations and the tasks space variables variations, accounting for the positional and rotational components of $T_{3 D}$, as presented by De Freitas and Scholz [50]. To validate the numerical estimation, for $T_{2 D}$, we computed the logarithmic ratio of the variances on both the analytical and numerical estimation of the Jacobian. For $T_{3 D}$, only the numerical computation was adopted.

1) Task $2 D\left(T_{2 D}\right)$ :The task space was planar $(x-y$ coordinates) with $d_{t a s k, T_{2 D}}=2$, and therefore, only the first two rows of the Jacobian are considered. The Jacobian matrix associated with this task can be expressed as follows:

$$
\mathbf{J}_{2 D, A}=\left[\begin{array}{cccc}
\frac{\partial \theta_{1}}{\partial x} & \frac{\partial \theta_{2}}{\partial x} & \ldots & \frac{\partial \theta_{d_{\text {joint }}}}{\partial x} \\
\frac{\partial \theta_{1}}{\partial y} & \frac{\partial \theta_{2}}{\partial y} & \ldots & \frac{\partial \theta_{d_{\text {joint }}}}{\partial y}
\end{array}\right]
$$

where the subscript $2 \mathrm{D}$ indicates the positional task space dimensions considered and A refers to the Analytical computation of the Jacobian.

In addition, the Jacobian associated with the positional task space (2D) was also estimated with the regression method $\mathbf{J}_{2 D, L S Q}$.

2) Task $3 \boldsymbol{D}\left(T_{3 D}\right)$ : In $T_{3 D}$, the elements of the Jacobian matrix were estimated with independent linear regressions 
between across-trial joint configurations and the endeffector tip positions and pitch angle (see Fig. 2B). Each row of the Jacobian matrix $\left(J_{3 D, L S Q}\right)$ was computed separately for each task space dimension and for each path-normalized sample as follows:

$$
\begin{aligned}
\Delta p x_{u, n, m, r}[s] & =\mathbf{J}_{x} \Delta \boldsymbol{\theta}_{u, n, m, r}[s] \\
\Delta p y_{u, n, m, r}[s] & =\mathbf{J}_{y} \Delta \boldsymbol{\theta}_{u, n, m, r}[s] \\
\Delta \alpha_{u, n, m, r}[s] & =\mathbf{J}_{\alpha} \Delta \boldsymbol{\theta}_{u, n, m, r}[s]
\end{aligned}
$$

Where $p x, p y$ and $\alpha$ are the user controlled task space variables, $J_{x}, J_{y}$ and $J_{\alpha}$ are the $1 \times 7$ separate rows of $\mathbf{J}_{3 D, L S Q}, \boldsymbol{\Delta} \boldsymbol{\theta}_{u, n, m, r}$ is the displacement from the mean joint configuration and $\{p x, p y, \alpha\}_{u, n, m, r}$ are the displacements from the task space variables for the $u^{t h}$ user, $n^{t h}$ task, $m^{t h}$ master device and $r^{t h}$ repetition. Under the assumption that the same arm state is defined for the same path position for each user and repetition, a system of ten equations corresponding to the 10 task repetitions can be defined for each line in Eq.12,13,14. Therefore, for each user, task, normalized frame and task space dimension, an overdetermined system of 10 equations with 7 unknowns has to be solved to obtain the corresponding Jacobian row. To improve the conditioning of the regression, the space-normalized signals were binned such that bins of 5 normalized samples were used to estimate each set of unknowns, thus solving systems of 50 equations with 7 unknowns using a Least Square Method (LSQ). Similarly, removing the $J_{\alpha}$ row, the LSQ method can be used to estimate the $J_{2 D, L S Q}$.

\section{H. Data Analysis}

1) Redundancy Exploitation: We used the $R_{v}$ value to measure the joint redundancy exploitation for each experiment and user in each normalized frame.

To analyze the possible modulation of the level of redundancy exploitation (HPI), and to test if the capability of efficiently partitioning joint variability in the task relevant and irrelevant manifolds was influenced by the path characteristics, we evaluated the $R_{v}$ along the normalized path. The trajectories of the two tasks were divided into eight parts, characterized by three levels of absolute curvature (Fig. 3.1) that were analytically obtained for each point along the bidimensional trajectories as in Buzzi et al. [13].

We used the Liellefors test $(\alpha=0.05)$ to evaluate the normality of the $R_{v}$ distributions for each normalized sample $[s]$ in each experiment among the seven users. Since the distributions were normal, the mean $R_{v}$ trajectories and the corresponding standard deviations in the four experimental conditions were obtained at each $s^{t h}$ space-normalized sample.

No significant trends were found in the $R_{v}$ throughout the path, and therefore, the $R_{v}$ mean values $\left(\bar{R}_{v u, n, m}\right)$ were extracted and grouped among users.

$$
\bar{R}_{v u, n, m}=\frac{1}{T_{n o r m}} \sum_{t=0}^{1} R_{v u, n, m}[s]
$$

To test differences between master devices (HP2) and tasks (HP3), as well as evaluating the differences between the two Jacobian computation methods, we fitted a 3-way repeatedmeasures ANOVA model with mean $R_{v}$ as dependent variable, task type, master device, and Jacobian computation method as fixed-effect within-user main independent factors; the interaction effects between the main factors were also evaluated. In this analysis, we compared the mean $R_{v}$ for all the experiments when a 3D task space is considered.

Focusing on $T_{3 D}$, the $\bar{R}_{v}$ was computed considering both the 2D positional task space $(x-y)$ and considering the additional tool orientation $(x-y-\alpha)$. To evaluate the differences in the $R_{v}$ introduced by the task space dimension, a follow up analysis was conducted on $T_{3 D}$. Using the LSQ Jacobian estimation method only, a 2-way repeated measures ANOVA was used to describe the data distributions in $T_{3 D}$ in which master device and task space dimension as well as their interaction are the fixed effect independent within users factors.

2) Error metrics: During the tasks execution, we evaluated the following metrics:

- Positional error $(d)$ : for both $T_{2 D}$ and $T_{3 D}$, we computed the planar Euclidean distance $(x-y)$ between the tool's end-effector tip and the closest point along the central line of the path (Fig. 1.A).

$$
d_{u, n, m, r}[s]=\sqrt{\begin{array}{l}
\left(p x_{u, n, m, r}[s]-t x_{u, n, m, r}[s]\right)^{2} \\
+\left(p y_{u, n, m, r}[s]-t y_{u, n, m, r}[s]\right)^{2}
\end{array}}
$$

With $t x$ and ty $x$ and $y$ coordinates of the closest point on the path center line in the $[s]$ space normalized frame.

- Angular error $(\psi)$ : for $T_{3 D}$ only, the angular displacement between the tool end effector pitch angle and the tangent to the path pitch angle in the closest point along the path was evaluated (Fig. 1.B).

$$
\psi_{u, n, m, r}[s]=p \alpha_{u, n, m, r}[s]-t \alpha_{u, n, m, r}[s]
$$

Where $t \alpha$ is the pitch angle of the tangent to the curve.

The normality of the distribution of $d$ and $\psi$ for each space normalized stamp among users and repetitions was tested (Liellefors test $\alpha=0.05$ ). Since the distributions were normal, the median $\bar{d}$ and $\bar{\psi}$ curves were extracted for each experiment.

To test the correlation between $R_{v}$ and the error metrics ( $d$ for $T_{2 D}$ and $T_{3 D}$ and $\psi$ for $T_{3 D}$ ) through the path, the Pearson linear correlation coefficient was computed for each experiment and user. Moreover, as a measure of the general correlation, we computed the Perason linear correlation between the mean $R_{v}$ and the mean position $(d)$ and orientation error $(\psi)$ throughout all the users acquired.

The mean $d$ and $\psi$ values among the ten repetitions and users were extracted for each experiment to test differences between master devices and tasks for $d$ and between master devices only for $\psi$. Therefore, for the positional error $d$ we fitted a 2-way repeated-measures ANOVA model with task type and master device as fixed effect within users independent factors. For the orientation error $\psi$, we used a 1-way repeatedmeasures ANOVA model with master device as fixed effect withing user independent factor. 


\section{Results}

\section{A. Redundancy Exploitation}

We first focused on the analysis of the redundancy exploitation associated with the bi-dimensional tool position only, for both tasks, regardless of differences in the task space dimension. Fig. 3 shows the $R_{v}$ modulation throughout the trajectories for the different master devices in the two tasks. For $T_{2 D}$, the $R_{v}$ was computed using $J_{2 D, A}$ and $J_{2 D, L S Q}$ (dashed line), and for $T_{3 D}$ only $J_{2 D, A}$ is presented. The color

A.
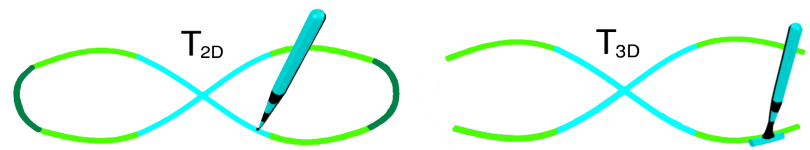

B.
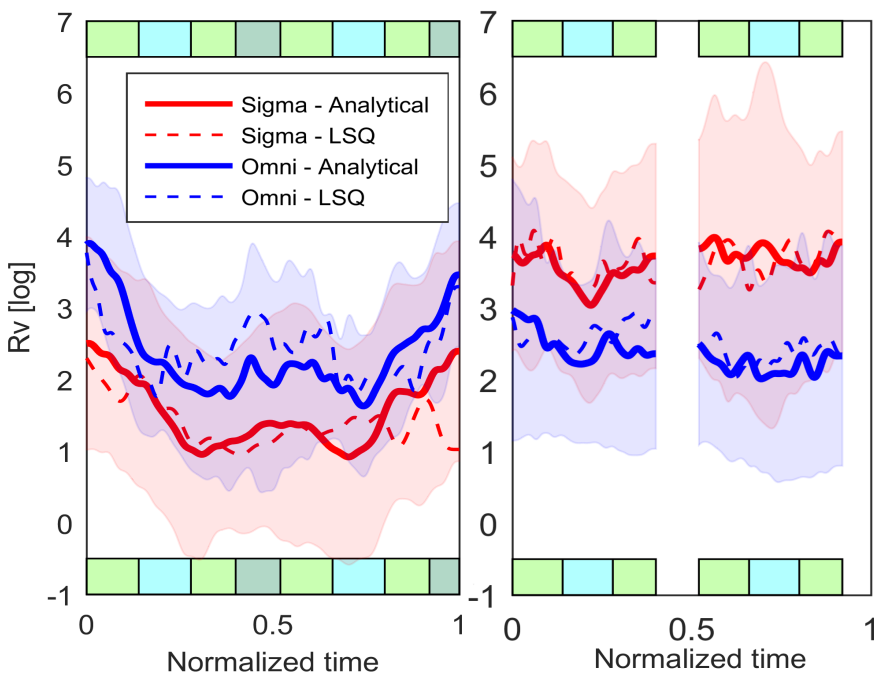

Fig. 3. (HP1) A. The curvature regions in the two tasks. B. Mean positional $\boldsymbol{R}_{v}$ and standard deviations through the trajectories while teleoperating with Sigma (in red) and Omni (in blue). The shaded regions represent the standard deviations.

bars at the top and bottom of the graphs correspond to the levels of curvature. Since the highest curvature part of the path (dark green) was missing from $T_{3 D}$, the corresponding parts of the graphs were left empty. A modulation of $R_{v}$ can be seen in $T_{2 D}$, where at the beginning and at the end of the path, users generally showed higher values with respect to other sections. Moreover, $R_{v}$ slightly increased in the central part of the path. On the other hand, in $T_{3 D}$, users did not show any noticeable change in their exploitation of the arm redundancy; in fact, in this case, the $R_{v}$ did not change throughout the path.

From the comparison between the two Jacobian computation methods that is presented in Fig. 3.B, in $T_{2 D}$, the LSQ method seems able to follow the Analytical computation within the variability range.

Fig. 4 shows the $R_{v}$ for $T_{2 D}$ and $T_{3 D}$ performed with the different master devices. The graph shows a comparison between the $R_{v}$ values for the positional task variables only (2D) obtained using the two Jacobian estimation methods: Analytical (A) and Least Square regression (LSQ). The results of the three-way ANOVA analysis performed are reported in Table I. Users showed higher $\bar{R}_{v}$ values associated with the
TABLE I

EFFECTS OF TASK, MASTER DEVICE AND JACOBIAN COMPUTATION METHOD ON $\overline{\boldsymbol{R}}_{\boldsymbol{v}}$ ASSOCIATED WITH THE POSITIONAL TASK DIMENSIONS

\begin{tabular}{lccc}
\hline Source & df & F & P \\
\hline Tasks & 1 & $\mathbf{1 2 . 0 6}$ & $\mathbf{0 . 0 0 0 9}$ \\
Master Devices & 1 & 2.10 & 0.154 \\
Jacobian type & 1 & 1.53 & 0.225 \\
Tasks*Master Devices & 1 & 1.09 & 0.303 \\
Tasks*Jacobian type & 1 & 0 & 0.988 \\
Master Devices*Jacobian type & 1 & 0.01 & 0.913 \\
Tasks*Master Devices*Jacobian type & 1 & 0.58 & 0.452 \\
Total & 54 & & \\
\hline
\end{tabular}

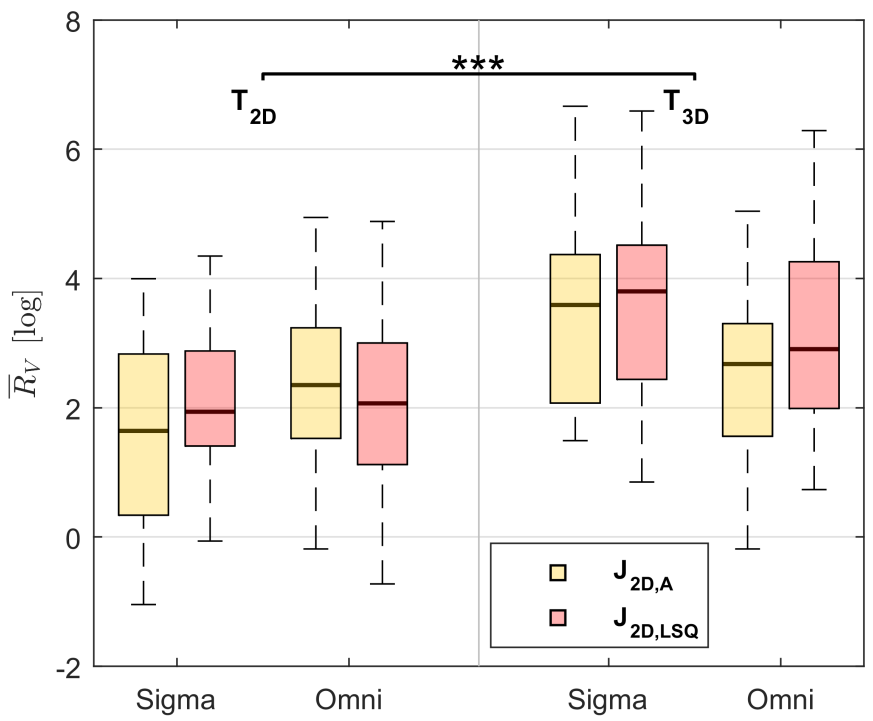

Fig. 4. (HP2 \& HP3) $\boldsymbol{R}_{\boldsymbol{v}}$ distribution among the experiments: $\boldsymbol{T}_{\mathbf{2}}$ and $\boldsymbol{T}_{3 D}$ performed with the hybrid parallel/serial link robot and with the serial link one, computed using the analytical Jacobian $\left(\boldsymbol{J}_{2 D, A}\right)$ and with the Least Squares Jacobian $\left(J_{2 D, L S Q}\right)$. The boxplots represent median values, first and third quartiles and minimal and maximal values. The statistical differences are depicted using lines and stars.

positional aspects of the task execution while performing the second task and this difference was statistically significant ( $p<0.001)$. No differences were found between the $R_{v}$ values obtained using the Analytical and the Least Square Jacobian estimation or between master devices.

In the second part of the analysis, we focused on $T_{3 D}$, studying the contribution of the tool end-effector orientation $(\alpha)$ to the redundancy exploitation. Fig. 5 shows the $\bar{R}_{v}$ distributions for $T_{3 D}$, computed considering the positional task space variables only (2D) and with the additional rotational task space variable (3D). When accounting for the rotational task space variable, users showed higher $\bar{R}_{v}$ values with respect to the $2 \mathrm{D}$ case, and this difference was significant $(p<0.05)$. Users showed roughly the same capability of exploiting the arm redundancy while teleoperating with the two master devices (Sigma/Omni) and no interaction effect was found either (see Table II).

\section{B. Error metrics}

Fig. 6 shows the mean positional error changes though the path for $T_{2 D}$ and $T_{3 D}$. The orientation error, described by 
TABLE II

EfFects of MASteR DEVICE AND TASK SPACE DIMENSION ON $\overline{\boldsymbol{R}}_{v}$ ASSOCIATED WITH $\boldsymbol{T}_{3 \boldsymbol{D}}$

\begin{tabular}{lccc}
\hline Source & df & F & P \\
\hline Master Devices & 1 & 0.01 & 0.910 \\
Task Space Dimension & 1 & $\mathbf{4 . 3 1}$ & $\mathbf{0 . 0 4 8}$ \\
Tasks Dimension *Master Devices & 1 & 0.67 & 0.420 \\
Total & 27 & & \\
\hline
\end{tabular}

$\psi$ is reported for $T_{3 D}$ only. For the positional error, a clear trend can be seen in $T_{2 D}$ for both master devices: the error decreases in correspondence with the high curvature parts of the path and increases when moving along the straight parts, almost symmetrically with respect to the normalized progression along the path. Similar behavior can be seen for the positional error in $T_{3 D}$, in which users showed minimal error when re-engaging the path after the open ends.

For $\psi$ in $T_{3 D}$, trends are less evident, but, as expected, the angular displacement between the end-effector direction and the tangent to the path was minimal on the straight portions of the path.

Table III shows the correlation indexes $(\rho)$ between $R_{v}$ and $d$ and $\psi$ in the four experiments among the users as well as the correlations between the mean $R_{v}$ values and the mean $d$ and $\psi$ curves. Although users' performance was characterized by different levels of correlation, the mean curves suggest that the positional error $(d)$ correlates negatively with the $R_{v}$ changes in $T_{2 D}$ for both master devices. Regarding $T_{3 D}$, both $\bar{d}$ and $\bar{\psi}$ showed very little correlation with the $\bar{R}_{v}$ modulation, but this is very likely due to the absence of modulation in the $R_{v}$ and error values in $T_{3 D}$.

Finally, regarding the positional error $d$, the ANOVA re-

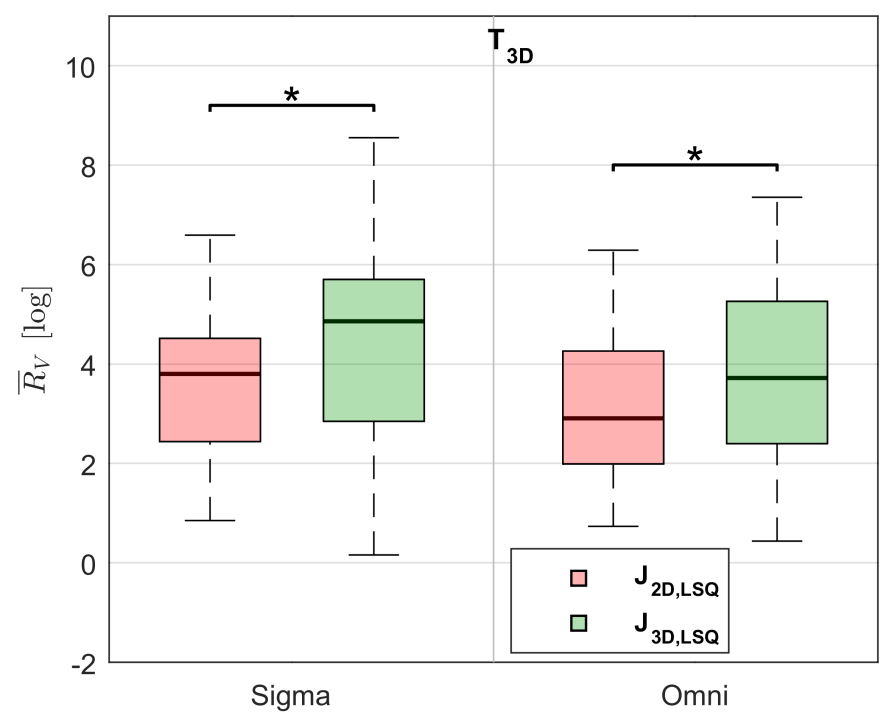

Fig. 5. (HP3) Differences in the $\boldsymbol{R}_{\boldsymbol{v}}$ distribution among users between different master devices and task space variables considered. In red the results obtained considering the positional only task variables $\left(\boldsymbol{J}_{2 D}\right)$ and in green when also the tool rotation is accounted $\left(\boldsymbol{J}_{3 D}\right)$. The boxplots represent median values, first and third quartiles and minimal and maximal values.

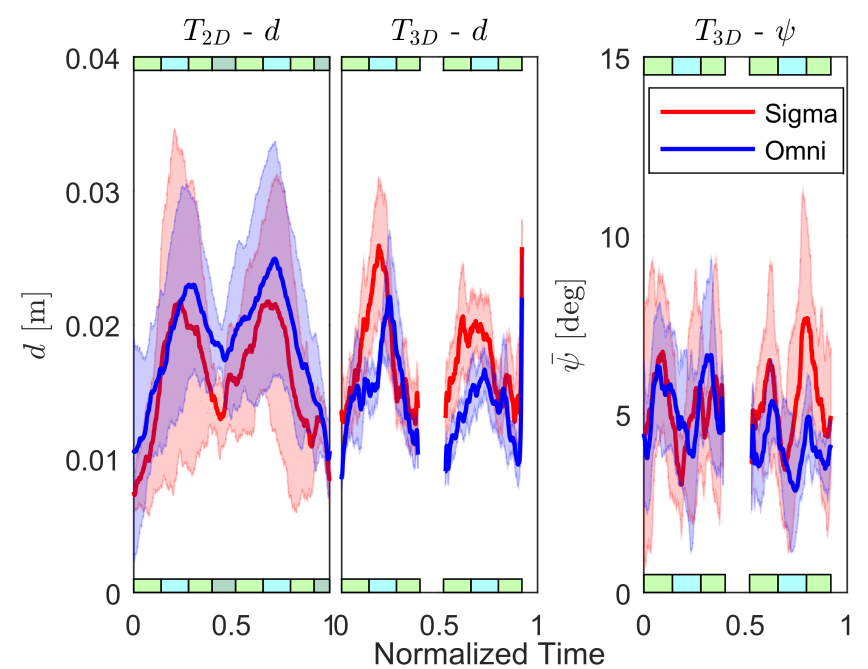

Fig. 6. Error metrics $\left(\boldsymbol{d}\right.$ and $\psi$ ) mean for $\boldsymbol{T}_{2 D}$ and $\boldsymbol{T}_{3 D}$. In red the hybrid parallel-serial link master device (Sigma) and in blue the serial link master device (Omni). The shaded regions represent the standard deviations.

TABLE III

PEARSON'S CORRELATIONS COEFFICIENTS BETWEEN $\boldsymbol{R}_{\boldsymbol{v}}$ AND $\boldsymbol{d}$ OR $\boldsymbol{\psi}$. ALL THE $\boldsymbol{\rho}$ ARE SIGNIFICANT $(\boldsymbol{p}<\mathbf{0 . 0 0 1})$

\begin{tabular}{rrrrrrr}
\hline & \multicolumn{2}{c}{$\boldsymbol{T}_{\mathbf{2 D}}-\boldsymbol{d}$} & \multicolumn{2}{c}{$\boldsymbol{T}_{\mathbf{3 D}}-\boldsymbol{d}$} & \multicolumn{2}{c}{$\boldsymbol{T}_{\mathbf{3 D}}-\psi$} \\
User & Sigma & Omni & Sigma & Omni & Sigma & Omni \\
\hline 1 & -0.18 & -0.46 & 0.35 & -0.14 & 0.35 & -0.09 \\
2 & -0.75 & 0.58 & -0.51 & 0.11 & -0.51 & 0.36 \\
3 & -0.18 & 0.11 & 0.07 & -0.26 & 0.07 & 0.14 \\
4 & -0.79 & -0.86 & -0.01 & -0.12 & -0.01 & 0.42 \\
5 & -0.52 & -0.14 & -0.22 & 0.18 & -0.22 & 0.30 \\
6 & -0.49 & -0.67 & -0.45 & 0.18 & -0.45 & -0.28 \\
7 & -0.85 & -0.77 & -0.13 & 0.21 & 0.20 & -0.16 \\
Median & -0.81 & -0.91 & -0.45 & -0.05 & 0.11 & 0.46 \\
\hline
\end{tabular}

sults showed no statistical difference between $T_{2 D}$ and $T_{3 D}$ $\left(F_{1,22}=1, p=0.33\right)$ nor between Sigma and Omni $\left(F_{1,22}=\right.$ $0.04, p=0.84$ ). In the same way, for $\psi$, the 1-way ANOVA showed no statistical difference between Sigma and Omni $\left(F_{1,22}=1.5, p=0.25\right)$.

\section{DISCUSSION}

We used the UCM analysis to investigate how different task constraints and master devices influence users' exploitation of arm redundancy. We found a significant modulation in the level of redundancy exploitation throughout the path in the positional task but less significant in the mixed positionorientation one. We used two Jacobian estimation methods (analytical and least square regression) and found that they were equally able to estimate the $R_{v}$ values in the positional task. During the execution of the position-orientation task, users achieved higher values of redundancy exploitation. Surprisingly, no significant differences were found between the two master devices tested in both tasks. We also analyzed task performance: no significant difference was found between the master devices, and users achieved the best performance in the positional task while maximizing the arm redundancy exploitation. 


\section{A. Redundancy Exploitation}

HP1: $R_{v}$ modulation - We first analyzed the $R_{v}$ modulation through the two tasks paths, and found a significant modulation of $R_{v}$ for $T_{2 D}$, but a constant level of redundancy exploitation for $T_{3 D}$. While no modulation in the redundancy exploitation was found for free-hand reaching movements [31], [48], more recent studies showed significant changes in the mean $R_{v}$ values with respect to the movement direction during planar teleoperated reaching task [34]. Nisky et al. observed a small reduction in the level of redundancy exploitation in the central part of reaching movements, and this trend is particularly clear for novices in free-hand and teleoperated task execution. Expert teleoperators, instead, showed flatter $R_{v}$ profiles, especially while teleoperating similarly to what was observed in $T_{3 D}$. However, the substantial differences between the traditional tasks and the eight-shaped path that we investigated in the current study, make it very difficult to compare our results with the existing literature. The task that we analyzed is more complex with respect to traditional reaching movements, and following each point along the path requires continuous changes in the direction of movement. Moreover, during the eight-shaped task execution, users relied on visual feedback, and the task required precision along the entire path, rather than just the endpoint in reaching. This required and allowed for on-line adjustments and corrections that are absent during faster goal-oriented reaching and pointing tasks.

The results of $T_{2 D}$, showed that users were maximizing the redundancy exploitation especially around the task's starting point rather than trying to compensate for the path's different movement directions or curvatures. It seems that, in $T_{2 D}$, users weren't capable of maintaining high levels of arm redundancy exploitation adapting to the different part of the path, instead, freezing to the initial kinematic solution, their redundancy exploitation performance decreased as they moved away from the starting point [51]. A reason that could explain this behavior can be found in the fact that, at the beginning of each repetition, users were able to freely reconfigure their arm position and orientation to adopt the kinematic solution that best fitted the starting position. As users moved away from the starting point, their capabilities in exploiting the arm redundancy decreased.

On the other hand, the $R_{v}$ modulation in $T_{3 D}$ is flatter: in this task, users were allowed to reconfigure their arm position as well as the end effector orientation after the first half of the path. This could have contributed to the increase in the users' capabilities in maximizing $R_{v}$. While performing $T_{3 D}$, users were able to maximize the ratio between the task irrelevant manifold and the task relevant manifold associated with the end-effector $\mathrm{x}-\mathrm{y}$ position to a higher extent with respect to $T_{2 D}$. On the other hand, analyzing the differences between the $\bar{R}_{v}$ profiles within the first half of the path, it is clear that the the possibility of reconfiguring the arm pose in correspondence with the path's open ends can't be the only factor that modified the users' redundancy exploitation. Reasons to explain the $\bar{R}_{v}$ flatness in the first half of $T_{3 D}$ must, therefore, lie in the task constraints and characteristics.
HP2: Differences between master devices - Regarding the hypothesis of differences in the $R_{v}$ modulation introduced by the two master devices architectures, apart from an offset between the two profiles, in both $T_{2 D}$ and $T_{3 D}$, Sigma and Omni allowed for very similar trends. This result suggests that the variation in the redundancy exploitation through the path isn't affected by the different master devices dynamics that are eventually even-out by the CNS. Looking at the mean distributions, the differences in the $R_{v}$ between the two master devices tested are non-significant. Nevertheless, trends can be seen in both tasks and, interestingly, while in $T_{2 D}$ the Omni master device shows higher values of $\bar{R}_{v}$, in $T_{3 D}$ the difference direction is opposite; in both cases, the differences in the mean values are hidden by large between users' variability. Previous studies showed that the manipulators' dynamic proprieties can influence performance and control strategies with respect to free-hand and unconstrained motion [34], [52]. Despite the mechanical and dynamical differences between Sigma and Omni, our results suggest that the differences in the kinematic solution adopted by users may require higher statistical power to be investigated. On the other hand, differences in the master devices could also be evened out by dynamic adaptation (as described in [13]) that would allow for similar $R_{v}$ modulations and mean values.

HP3: Influence of task constraints - We showed that no significant differences were found between the mean $R_{v}$ estimated with the Analytical Jacobian $\left(J_{2 D, A}\right)$ and the one obtained with the least square regression $\left(J_{2 D, L S Q}\right)$ when the same task space dimension is compared (x-y end-effector position). This result confirms that the two Jacobian estimations are similar [50].

Significant differences between task space dimensions were found in the mean $R_{v}$ in $T_{3 D}$ when the end-effector orientation is considered in the task space. $J_{3 D, L S Q}$ is therefore capable of accounting for the additional portion of joint variability that users were employing to minimize the angular displacement between the tool end-effector direction and the tangent to the path. Similar results were observed in pistol shooting [38] and golf swing [39] experiments: users were utilizing part of the joint variability to finely control the tools orientation rather than its sole position. On the other hand, contrary to previous studies [39], [41], the level of redundancy exploitation is constant through the task execution. This is likely because our tracing task requires a continuous control over the tool orientation, whereas golf and table-tennis players must coordinate their arm kinematics especially when the club/racket hits the ball.

Comparing the $\bar{R}_{v}$ between $T_{2 D}$ and $T_{3 D}$, it appears that users were able to elicit higher levels of redundancy exploitation when performing the most challenging task. This surprising result may have several explanation: it is possible that users, being more challenged by the second task, were also more inclined to explore a higher number of possible kinematic solutions. This difference in the level of task redundancy exploitation could be also related with the higher intrinsic motivation that users experience when challenged with a more 
complex motor task [53]-[55].

\section{B. Error metrics}

The results of the error metric analysis showed that users performance changed through the path. These changes are especially evident in the positional error, that shows similar behaviors for both master devices. Similarly to $R_{v}$, the positional error increases while moving away from the initial point, reaching its peaks in the straight and low curvature parts of the path. Regarding the orientation error $(\psi)$, as expected, users committed the smallest errors when teleoperating in the straight parts of the task where the changes in the tangent to the path are limited.

Interestingly, no significant differences were found between the errors, both positional and orientational, that users committed using the hybrid parallel-serial master device (Sigma) and the serial one (Omni). Although being characterized by significantly different structures, dynamics and costs, the users' performance with the two master devices are comparable. A reason for this could be found in the fact that the tasks studied weren't complex enough to stress the capabilities of each master device.

The results of the correlation analysis showed that different users adopted different strategies to limit the positional and orientational error, both for $T_{2 D}$ and $T_{3 D}$. On the other hand, looking at the correlations between mean $R_{v}$ and mean $d$, it seems that, regardless of the master device used, when users showed the maximal redundancy exploitation, they also committed the smallest positional errors. The same ability to increase the ratio between TIM and TRM without deteriorating task performance was observed also in expert stone knappers [56], music players [57] and golfers [58]. This trend, which is particularly evident in $T_{2 D}$, is absent in $T_{3 D}$. In this case, the lack of variation in $R_{v}$, as well as in the performance metrics may have limited the capabilities of finding correlations. Moreover, the mixed positional and orientational constraint introduced in $T_{3 D}$ may have influenced the strategies that users adopted during teleoperation, leading to more complex relations between the task space performance metrics and the redundancy exploitation.

The strong correlation between performance and arm redundancy exploitation is a clear sign of the importance of this motor property: advantageous master devices should not limit or modify arm kinematics. On the other hand, modern teleoperation consoles, such as the one adopted in the Da Vinci Robot, employ arm rests to increase user comfort and reduce muscular fatigue. While laying the forearm on the armrest, surgeons might limit their capabilities in finding the arm configuration that would allow for the maximization of the ratio between task unrelated and task related variability. Future studies are needed to consider the possible tradeoffs in using armrests, including fixed and dynamic support [59]

\section{Limitations and future work}

An important limitation of the UCM approach is the coordinate sensitivity of the computational procedures that define the task relevant and irrelevant manifolds [29]. The structure of the observed variability is defined in a coordinate space which is arbitrarily selected by the researcher among multiple others. For example, in arm joint variability, choosing absolute or relative angles to describe the joints can yield to different results. When the structure of variability is evaluated in the result space defined by the task, i.e. using the TNC analysis, a sensitivity to changes in the coordinates frame exists, but is far less severe [29].

To solve these issues, a coordinate-independent formulation of the UCM analysis was recently proposed [30]. Campolo et al. exploited a particular type of kinetic energy metric originally proposed in [60] to study the level of redundancy exploitation during a static pointing task performed with an hand-handled tool. The choice of a left-invariant metric, which is independent from the frames used to describe the tool kinematics, allowed to estimate a coordinate-independent level of redundancy exploitation in a static task. This successful extension of the UCM analysis was applied to date to static tasks only, and future studies are needed to adopt this promising framework to the analysis of dynamic tasks such as following a path (tracing) or a trajectory (tracking).

In the present work, we performed a comparative analysis of different tasks and conditions, with the aim of underling the effects of different master interfaces and tasks constraints rather than striving to obtain absolute estimations of the level of redundancy exploitation. We, focused on expanding the existing literature regarding the effects of mixed positional/orientational constraints over arm redundancy, exploiting analytical techniques that have been already employed for non-static tasks [61], [62]. While coordinate-dependency still represents a non-negligible limitation of the present work, the comparative nature of the analysis should make it less sensitive to changes in the joints reference frames. Future studies are needed to develop a coordinate-independent metric similar to [30] that would be appropriate for our current task and for future studies with surgical tasks.

Another limitation of our study is its power. The number of users that took part in the experiments was small which reduced the statistical power of some of the analysis conducted. Further studies should therefore focus on extending the analysis to a higher number of participants.

The absence of differences in terms of both performance and redundancy exploitation between the master devices tested should be further analyzed. First, in order to study how users cope with substantially different kinematics, ergonomics and workspaces, it would be interesting to extend the analysis to other master devices (i.e. the Da Vinci master console). Moreover, it would be interesting to evaluate how the use of an arm rest would affect users capability of exploiting arm redundancy. Secondly, the introduction of more complex tasks, characterized by higher task space dimensions, would allow to stress the effects of the different kinematic and dynamic characteristics on the motor strategies adopted by the users. The methods adopted in the present work could also be used to estimate how the additional task space dimensions would modify the users' redundancy exploitation.

Additionally, in the present work, no differences were found among users, who were all novice teleoperators. Previous 
studies showed how experienced and novice users adopt different arm redundancy exploitation strategies. Therefore, it would be interesting to study how users with different levels of familiarity and skills in teleoperation would cope with differences in the task constraint and task space dimensions. Moreover, the possibility of having multiple experimental sessions for each user, would allow to apply the UCM analysis to study how the level of redundancy exploitation changes throughout the learning process.

\section{CONCLUSION}

We used the UCM analysis to explore the differences induced by diverse task space dimensions and constraints and two master devices on teleoperators capabilities on the exploitation of the arm redundancy. We validated different algorithms to estimate the Jacobian matrix associated with the motion, and found significant differences between different tasks space dimensions. We found that users maximized the ratio between task irrelevant and task relevant variability especially in the more complex task, characterized by positional and rotational task space variables, regardless of the mechanical differences in the type of master device. Moreover, during the execution of the 2D task, users showed the lowest positional error while maximizing their arm redundancy, showing a positive correlation between performance and redundancy exploitation.

The present work represents a first step towards an in depth understanding of the mechanisms that regulate motor control during the execution of complex teleoperation tasks. The results showed how, for the tasks studied, substantially different master devices led to similar outcomes both in terms of performance and arm redundancy exploitation and how teleoperators can take advance of an additional portion of arm redundancy during mixed positional-orientational tasks.

\section{ACKNOWLEDGMENT}

This project has received funding from the European Union's Horizon 2020 research and innovation programme under grant agreement No. H2020-ICT-2016-732515 and from the Helmsley Charitable Trust through the ABC Robotics Initiative, by the Marcus Endowment Fund both at Ben-Gurion University of the Negev, and the ISF grant number 823/15

\section{REFERENCES}

[1] Y.-Y. Juo, A. Mantha, A. Abiri, A. Lin, and E. Dutson, "Diffusion of robotic-assisted laparoscopic technology across specialties: a national study from 2008 to 2013," Surgical Endoscopy, pp. 1-9, 2017.

[2] R. D. Howe and Y. Matsuoka, "Robotics for surgery," Annual Review of Biomedical Engineering, vol. 1, no. 1, pp. 211-240, 1999.

[3] B. Johnson and G. Somu, "Robotic Telesurgery: Benefits Beyond Barriers," BMH Medical Journal, vol. 3, no. 2, 2016.

[4] A. R. Lanfranco, A. E. Castellanos, J. P. Desai, and W. C. Meyers, "Robotic surgery: a current perspective," Annals of surgery, vol. 239, no. 1, pp. 14-21, 2004.

[5] N. Enayati, G. Ferrigno, and E. De Momi, "Performance metrics for guidance active constraints in surgical robotics," The International Journal of Medical Robotics and Computer Assisted Surgery, vol. 14, no. $1,2018$.

[6] T. Zhou, M. E. Cabrera, T. Low, C. Sundaram, and J. Wachs, "A comparative study for telerobotic surgery using free hand gestures," Journal of Human-Robot Interaction, vol. 5, no. 2, pp. 1-28, 2016.
[7] H. Jiang, B. S. Duerstock, and J. P. Wachs, "User-centered and analyticbased approaches to generate usable gestures for individuals with quadriplegia," IEEE Transactions on Human-Machine Systems, vol. 46, no. 3, pp. 460-466, 2016.

[8] E. Beretta, F. Nessi, G. Ferrigno, F. D. Meco, A. Perin, L. Bello, G. Casaceli, F. Raneri, A. D. Benedictis, and E. D. Momi, "Enhanced torque-based impedance control to assist brain targeting during openskull neurosurgery: a feasibility study," The International Journal of Medical Robotics and Computer Assisted Surgery, vol. 12, no. 3, pp. 326-341, jul 2015.

[9] I. Nisky, A. M. Okamura, and M. H. Hsieh, "Effects of robotic manipulators on movements of novices and surgeons," Surgical endoscopy, vol. 28, no. 7, pp. 2145-2158, 2014.

[10] N. Enayati, E. De Momi, and G. Ferrigno, "Haptics in robot-assisted surgery: challenges and benefits," IEEE reviews in biomedical engineering, vol. 9, pp. 49-65, 2016.

[11] N. Enayati, G. Ferrigno, and E. De Momi, "Skill-based human-robot cooperation in tele-operated path tracking," Autonomous Robots, pp. 113, 2017.

[12] M. L. Latash, J. P. Scholz, and G. Schöner, "Motor control strategies revealed in the structure of motor variability," Exercise and sport sciences reviews, vol. 30, no. 1, pp. 26-31, 2002.

[13] J. Buzzi, J. Jansma, G. Ferrigno, and E. De Momi, "On the value of estimating human arm stiffness during virtual teleoperation with robotic manipulators," Frontiers in Neuroscience, vol. 11, p. 528, 2017.

[14] J. Buzzi, C. Gatti, G. Ferrigno, and E. De Momi, "Analysis of joint and hand impedance during teleoperation and free-hand task execution," IEEE Robotics and Automation Letters, vol. 2, no. 3, pp. 1733-1739, 2017.

[15] M. L. Latash, "The Bernstein problem: how does the central nervous system make its choices," Dexterity and its development, pp. 277-303, 1996.

[16] M. Kawato, Y. Maeda, Y. Uno, and R. Suzuki, "Trajectory formation of arm movement by cascade neural network model based on minimum torque-change criterion," Biological cybernetics, vol. 62, no. 4, pp. 275288, 1990.

[17] P. Viviani and T. Flash, "Minimum-jerk, two-thirds power law, and isochrony: converging approaches to movement planning." Journal of Experimental Psychology: Human Perception and Performance, vol. 21, no. 1, p. 32, 1995.

[18] B. I. Prilutsky and V. M. Zatsiorsky, "Optimization-based models of muscle coordination," Exercise and sport sciences reviews, vol. 30, no. 1, p. 32, 2002.

[19] O. Sporns and G. M. Edelman, "Solving Bernstein's problem: A proposal for the development of coordinated movement by selection," Child development, vol. 64, no. 4, pp. 960-981, 1993.

[20] E. Todorov, "Interpreting motor adaptation results within the framework of optimal feedback control," Advances in Computational Motor Control, vol. 1, 2002.

[21] E. Todorov and M. I. Jordan, "Optimal feedback control as a theory of motor coordination," Nature neuroscience, vol. 5, no. 11, p. 1226, 2002.

[22] I. M. Gelfand and M. L. Latash, "On the problem of adequate language in motor control," Motor control, vol. 2, no. 4, pp. 306-313, 1998.

[23] M. L. Latash, "The bliss (not the problem) of motor abundance (not redundancy)," Experimental brain research, vol. 217, no. 1, pp. 1-5, 2012.

[24] H. Müller and D. Sternad, "Decomposition of variability in the execution of goal-oriented tasks: three components of skill improvement." Journal of Experimental Psychology: Human Perception and Performance, vol. 30, no. 1, p. 212, 2004.

[25] J. P. Cusumano and P. Cesari, "Body-goal variability mapping in an aiming task," Biological cybernetics, vol. 94, no. 5, pp. 367-379, 2006.

[26] J. B. Dingwell, R. F. Smallwood, and J. P. Cusumano, "Trial-to-trial dynamics and learning in a generalized, redundant reaching task," Journal of Neurophysiology, vol. 109, no. 1, pp. 225-237, 2013.

[27] J.-F. Yang, J. P. Scholz, and M. L. Latash, "The role of kinematic redundancy in adaptation of reaching," Experimental brain research, vol. 176, no. 1, pp. 54-69, 2007.

[28] F. J. Valero-Cuevas, M. Venkadesan, and E. Todorov, "Structured variability of muscle activations supports the minimal intervention principle of motor control," Journal of neurophysiology, vol. 102, no. 1, pp. 5968, 2009.

[29] D. Sternad, S.-W. Park, H. Müller, and N. Hogan, "Coordinate dependence of variability analysis," PLoS computational biology, vol. 6, no. 4, p. e1000751, 2010. 
[30] D. Campolo, F. Widjaja, H. Xu, W. T. Ang, and E. Burdet, "Analysis of accuracy in pointing with redundant hand-held tools: a geometric approach to the uncontrolled manifold method," PLoS computational biology, vol. 9, no. 4, p. e1002978, 2013.

[31] D. Domkin, J. Laczko, S. Jaric, H. Johansson, and M. L. Latash, "Structure of joint variability in bimanual pointing tasks," Experimental brain research, vol. 143, no. 1, pp. 11-23, 2002.

[32] R. Rein, B. Bril, and T. Nonaka, "Coordination strategies used in stone knapping," American Journal of Physical Anthropology, vol. 150, no. 4, pp. 539-550, 2013.

[33] M. L. Latash, J. F. Scholz, F. Danion, and G. Schöner, "Structure of motor variability in marginally redundant multifinger force production tasks," Experimental brain research, vol. 141, no. 2, pp. 153-165, 2001.

[34] I. Nisky, M. H. Hsieh, and A. M. Okamura, "Uncontrolled manifold analysis of arm joint angle variability during robotic teleoperation and freehand movement of surgeons and novices," IEEE Transactions on Biomedical Engineering, vol. 61, no. 12, pp. 2869-2881, 2014.

[35] I. Nisky, Y. Che, Z. F. Quek, M. Weber, M. H. Hsieh, and A. M. Okamura, "Teleoperated versus open needle driving: Kinematic analysis of experienced surgeons and novice users," in Robotics and Automation (ICRA), 2015 IEEE International Conference on. IEEE, 2015, pp. 5371-5377.

[36] Y. Sharon, T. Lendvay, and I. Nisky, "Instrument tip angular kinematics in teleoperated needle-driving," in Proc. Hamlyn Symposium on Medical Robotics, 2016, pp. 96-97.

[37] Y. Sharon, T. S. Lendvay, and I. Nisky, "Instrument orientation-based metrics for surgical skill evaluation in robot-assisted and open needle driving," arXiv preprint arXiv:1709.09452, 2017.

[38] J. P. Scholz, G. Schöner, and M. L. Latash, "Identifying the control structure of multijoint coordination during pistol shooting," Experimental Brain Research, vol. 135, no. 3, pp. 382-404, 2000.

[39] A. Morrison, D. McGrath, and E. S. Wallace, "Motor abundance and control structure in the golf swing," Human movement science, vol. 46, pp. 129-147, 2016.

[40] T. Flash and F. Mussa-Ivaldi, "Human arm stiffness characteristics during the maintenance of posture," Experimental Brain Research, vol. 82, no. 2, pp. 315-326, 1990.

[41] Y. Iino, S. Yoshioka, and S. Fukashiro, "Uncontrolled manifold analysis of joint angle variability during table tennis forehand," Human movement science, vol. 56, pp. 98-108, 2017.

[42] N. Levit-Binnun, E. Schechtman, and T. Flash, "On the similarities between the perception and production of elliptical trajectories," $E x$ perimental brain research, vol. 172, no. 4, pp. 533-555, 2006.

[43] R. Horaud and F. Dornaika, "Hand-eye calibration," The international journal of Robotics Research, vol. 14, no. 3, pp. 195-210, 1995.

[44] R. A. Prokopenko, A. A. Frolov, E. V. Biryukova, and A. Roby-Brami, "Assessment of the accuracy of a human arm model with seven degrees of freedom," Journal of biomechanics, vol. 34, no. 2, pp. 177-185, 2001.

[45] K. R. Saul, X. Hu, C. M. Goehler, M. E. Vidt, M. Daly, A. Velisar, and W. M. Murray, "Benchmarking of dynamic simulation predictions in two software platforms using an upper limb musculoskeletal model," Computer methods in biomechanics and biomedical engineering, vol. 18 , no. 13, pp. 1445-1458, 2015.

[46] K. R. S. Holzbaur, W. M. Murray, and S. L. Delp, "A model of the upper extremity for simulating musculoskeletal surgery and analyzing neuromuscular control," Annals of biomedical engineering, vol. 33, no. 6, pp. 829-840, 2005.

[47] S. L. Delp, F. C. Anderson, A. S. Arnold, P. Loan, A. Habib, C. T. John, E. Guendelman, and D. G. Thelen, "OpenSim: open-source software to create and analyze dynamic simulations of movement," IEEE Transactions on Biomedical Engineering, vol. 54, no. 11, pp. 19401950, 2007.

[48] J. P. Scholz and G. Schöner, "The uncontrolled manifold concept: identifying control variables for a functional task," Experimental brain research, vol. 126, no. 3, pp. 289-306, 1999.

[49] M. L. Latash, J. P. Scholz, and G. Schöner, "Toward a new theory of motor synergies," Motor control, vol. 11, no. 3, pp. 276-308, 2007.

[50] S. M. S. F. de Freitas and J. P. Scholz, "A comparison of methods for identifying the Jacobian for uncontrolled manifold variance analysis," Journal of biomechanics, vol. 43, no. 4, pp. 775-777, 2010.

[51] B. Steenbergen, R. G. Marteniuk, and L. E. Kalbfleisch, "Achieving coordination in prehension: joint freezing and postural contributions," Journal of motor behavior, vol. 27, no. 4, pp. 333-348, 1995.

[52] M. Desmurget, M. Jordan, C. Prablanc, and M. Jeannerod, "Constrained and unconstrained movements involve different control strategies," Journal of neurophysiology, vol. 77, no. 3, pp. 1644-1650, 1997.
[53] M. H. Anshel, R. Weinberg, and A. Jackson, "The effect of goal difficulty and task complexity on intrinsic motivation and motor performance," Journal of Sport Behavior, vol. 15, no. 2, p. 159, 1992.

[54] M. Goudas, K. Minardou, and I. Kotis, "Feedback regarding goal achievement and intrinsic motivation," Perceptual and Motor Skills, vol. 90, no. 3, pp. 810-812, 2000.

[55] G. P. Latham, E. A. Locke, and N. E. Fassina, "The high performance cycle: Standing the test of time," Psychological management of individual performance, pp. 201-228, 2002.

[56] V. Roux, B. Bril, and G. Dietrich, "Skills and learning difficulties involved in stone knapping: The case of stone-bead knapping in khambhat, india," World Archaeology, vol. 27, no. 1, pp. 63-87, 1995.

[57] J. Verrel, S. Pologe, W. Manselle, U. Lindenberger, and M. Woollacott, "Coordination of degrees of freedom and stabilization of task variables in a complex motor skill: expertise-related differences in cello bowing," Experimental brain research, vol. 224, no. 3, pp. 323-334, 2013.

[58] P. Glazier, "Movement variability in the golf swing: Theoretical, methodological, and practical issues," Research quarterly for exercise and sport, vol. 82, no. 2, pp. 157-161, 2011.

[59] M. A. Fehlberg, I. Nisky, A. J. Doxon, and W. R. Provancher, "Improved active handrest performance through use of virtual fixtures," IEEE Transactions on Human-Machine Systems, vol. 44, no. 4, pp. 484-498, 2014.

[60] F. C. Park, "Distance metrics on the rigid-body motions with applications to mechanism design," Journal of Mechanical Design, vol. 117, no. 1, pp. 48-54, 1995.

[61] D. S. Reisman, J. P. Scholz, and G. Schöner, "Differential joint coordination in the tasks of standing up and sitting down," Journal of Electromyography and Kinesiology, vol. 12, no. 6, pp. 493-505, 2002.

[62] T. Bennett, C. Low, and C. Cooke, "Instep soccer kick technique and performance through an analysis of movement varlabilln," in ISBSConference Proceedings Archive, vol. 34, no. 1, 2016. 